\title{
SPACE DISTRIBUTION OF SHORT PERIOD MIRA VARIABLES
}

\section{J. HRON}

Institut für Astronomie der Universität Wien, Türkenschanzstraße 17, A-1180 Wien, Austria

The group of short period Mira variables (periods less than 200 days) is thought to be a mixture of metal poor stars and disk stars. We have compiled JHKL photometry for 159 stars in this period range, the great majority of the data coming from new observations. The photometry is used to separate metal poor from disk stars, following Hron (1991, A\&A 252, 583), and to define volume limited subsamples. Distances were derived from the $K$-magnitudes and the period/ $K$-luminosity relationship.

The mean distances from the galactic plane for the two metallicity groups confirm our photometric metallicity criteria (Fig. 1). We present first results on the scale heights and number densities of the metal poor and disk stars. These parameters were determined by two different methods which give slightly, but systematically, different values. The disk stars have a scale height comparable to that of Miras with longer periods. The scale height of the metal poor stars is about $1 \mathrm{kpc}$. Thus both the metallicity and scale height of this group of stars are typical for stars in the galactic thick disk, although the galactic kinematics apparently are differen$\mathrm{t}$ from thick disk stars. The value of the scale height and its independence from galactocentric distance strongly favour a flattened space distribution, rather than a spherical one.

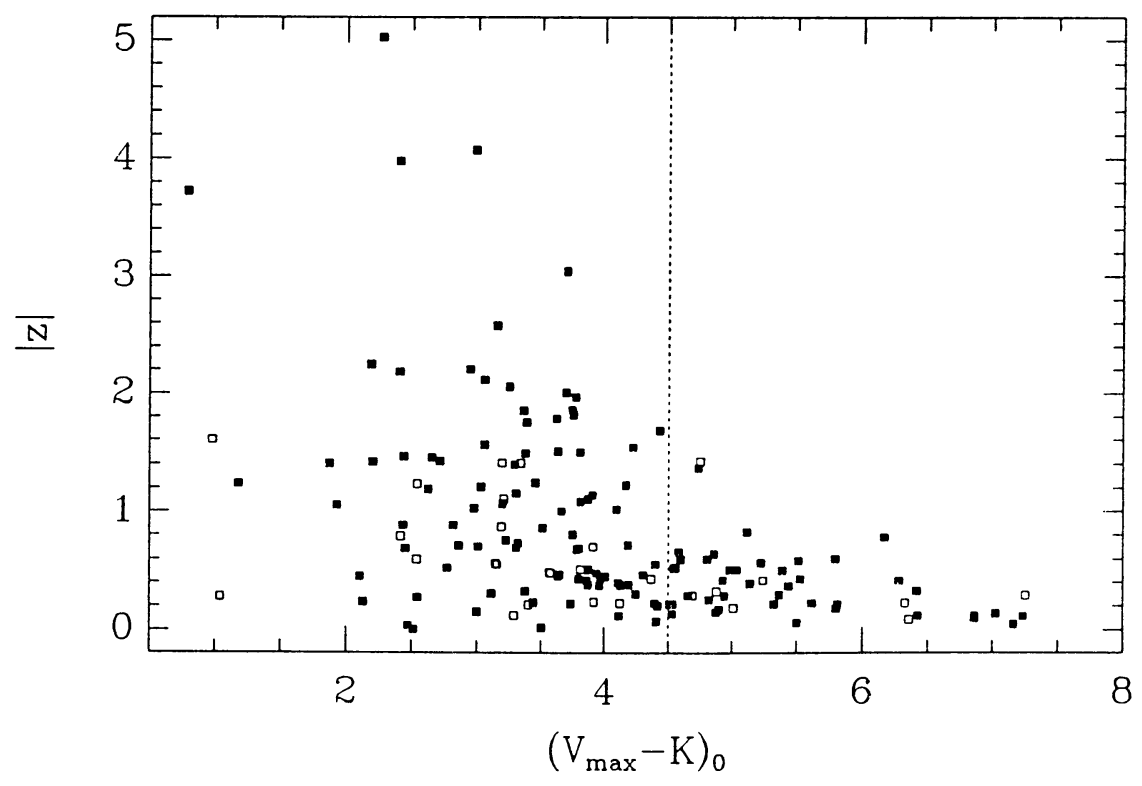

Fig. 1. $\left(V_{\max }-K\right)_{0}$ versus distance from the galactic plane. Open squares are stars with $P<140^{\mathrm{d}}$. 